\title{
Nonlinear Modeling Method Based on RBF Neural Network Trained by AFSA with Adaptive Adjustment
}

\author{
Xu-Sheng Gan ${ }^{1, a}$, Zhi-bin Chen ${ }^{2, b}$,Ming-gong Wu ${ }^{2, c}$ \\ ${ }^{1}$ XiJing College, Xi’an, Shaanxi, 710123, China \\ ${ }^{2}$ Air Traffic Control and Navigation College, Air Force Engineering University, Xi’an, Shaanxi, 710051, \\ China \\ a ganxusheng123@163.com, ${ }^{b}$ zhibin_chen@sina.com, ${ }^{c}$ mg_w321@163.com
}

Keywords: Radial basis function; Neural network; Artificial fish swarm algorithm; Nonlinear function

\begin{abstract}
To improve the nonlinear modeling capability of RBF neural network, an Artificial Fish Swarm Algorithm (AFSA) training algorithm with an adaptive mechanism is proposed. In the training algorithm, the search step size and visible domain of AFSA algorithm can be adjusted dynamically according to the convergence characteristics of artificial fish swarm, and then the improved AFSA algorithm is used to optimize the parameters of RBF neural network. The example shows that, the proposed model is a better approximation performance for the nonlinear function.
\end{abstract}

\section{Introduction}

Radial Basis Function (RBF) neural network is an abstraction simulation of brain neural network. and shows a satisfactory modeling effect with good convergence. Presently, the algorithms of RBF neural network mainly have the orthogonal least square, hybrid recursive, RAN, etc.. In recent years, the intelligent algorithms are also present [1][2].

In the paper, Artificial Fish Swarm Algorithm (AFSA) is introduced to train RBF network for the improvement of performance. The experiment shows the feasibility and efficiency of the algorithms.

\section{Adaptive artificial fish swarm algorithm}

AFSA first proposed by Li Xiaolei et al in 2001, is a novel swarm intelligence optimization algorithm that simulates the foraging behavior of fish swarm. Its basic principle is to learn from the foraging, clustering and pileup behavior of fish, start from the bottom behavior of the single artificial fish, through local optimization of each individual in fish swarm, reaches the purpose that the global optimum value emerges in the swarm. Once the algorithm appears, the algorithm has pay attention, and puts forward some improved AFSA algorithm, is widely used in pattern recognition, optimizing parameters in the field [3][4].

The foraging behavior laid the foundation of the convergence of the algorithm; the clustering behavior can improve the stability and global performance of the algorithm convergence; the pileup behavior can enhances speed and global performance of the algorithm convergence. The evaluation $\mathrm{f}$ its behavior also provides the guarantee for the speed and stability of the algorithm convergence. In general, the value range of each parameter in the algorithm was very tolerant without the requirement of the initial value of the algorithm.

In view of the artificial fish model described above and its behavior, each artificial fish explore the environments at present (including: the change of the partners and the objective function), so as to choose a behavior, and ultimately, the artificial fishes gather around several local extremum. Normally, for solving the problem of maximum problem, the artificial fish with larger food concentration value is around the extreme value range that the target value is larger, which helps to 
acquire the global extremum range; and generally more artificial fishes gather around the extreme value range that the target value is larger, which helps to judge and get the global extremum.

In foraging behavior of artificial fish, the individual always try to move toward a better direction, which laid the foundation of the convergence of the algorithm. The artificial fish randomly inspect the state $X_{j}$ of a point in the range of its vision. If it is better than the current state $X_{i}$, move forward one step along the direction of the state $X_{i}$ to reach the state $X_{j}$; if the state $X_{j}$ is not better than the state $X_{i}$, it continues to randomly inspect the state within the range of its vision, if the number of inspections reach a certain number of times try-number , and has not yet found a better state, perform the random walk. In this process, the step size Step and the visible domain Visual have a great influence on the speed and precision of convergence of fish swarm algorithm. Set improperly it to fall into a local minimum or not up to the precision. In foraging behavior, if the individual of artificial fish did not find a better state, a new state is randomly chosen without making full use of the information obtained, resulting in the increasing computation and slow convergence.

The change of visual domain have great influence on the algorithm convergence. As Visual is smaller, the foraging behavior and random walk of the artificial fish is more prominent, the local search ability of the algorithm is strong; as Visual is larger, the pileup behavior and clustering behavior of the artificial fish is more prominent, the global search ability and fast convergence of the algorithm is strong. In addition, the infleunce of Step can not be ignored, Step is large, the convergence is fast, but occasionally there are the oscillations; Step is small, the convergence is slow, but the accuracy is very high.

From the analysis, more difficult the function is optimized, stronger the the global search ability is required. Once the approximately optimal position is located, it is required to improve the local search ability and strengthen the fine search. Therefore, the method that dynamically adjust Visual and Step can be used to improve the performance of AFSA algorithm. At the early stage of the algorithm, the larger Visual and Step can be used to enhance the global search capability and convergence speed of the algorithm, in order that the artificial fish can perform the coarse search within a large range, with the progression of the search, Visual and Step are gradually reduced. At the late stage of the algorithm, the global search at the early stage is gradually translated into the local search, and located and searched near the optimal solutions, it can improve the local search ability and accuracy of optimization algorithm. Visual and Step can be adjusted dynamically by

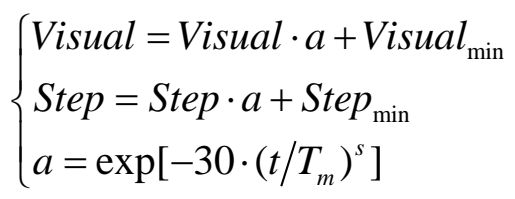

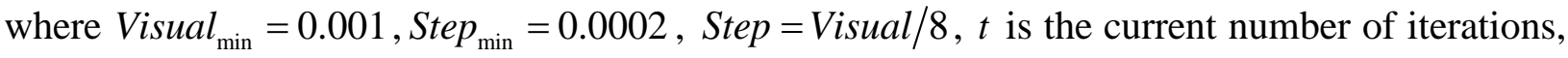
$T_{\mathrm{m}}$ is the maximum number of iterations. Usually the initial value Visual is $Z_{\mathrm{m}} / 4\left(Z_{\mathrm{m}}\right.$ is the maximum value of the search range). The Visual and Step function is composed of 3 stages, at the early stage of the algorithm, keep the maximum value, and then gradually change from large to small, finally keep the minimum value. This method is a good balance between the global search capability and local search capability, speeds up the convergence, improves the precision of the algorithm. The change rate $s$ of the value of the function $a$ from large to small is an integer greater than l, usually the values range is $[1,30]$. Fig. 1 shows the variation curve of the function $a$ when $T_{\mathrm{m}}=1000$ and $s$ is taken as $3,6,12$ respectively.

\section{Brief introduction of RBF neural network}

RBF neural network can approximate any nonlinear function and process the regularity that is difficult of the analysis in the system, and has good generalization ability with fast convergence speed. 
At present, it has been successfully applied in the domain such as nonlinear function approximation, time series analysis, data classification, pattern recognition, information processing, image processing, system modeling, control and fault diagnosis and so on.

The convergence of RBF neural network learning is fast. When one or more adjustable parameters of the network (the weights or thresholds) have an effect on any output, such a network can be called the global approximation network. For each input, each weight value of the network need to be adjusted, so as to leads to the slow learning speed of global approximation network. BP neural network is a typical example. If there are only a few connection weights affected the output for a certain local region in the input space, then the network can be called as the local approximation network. familiar local approximation network includes: RBF network, cerebellar model articulation controller (CMAC) network, B spline network etc..

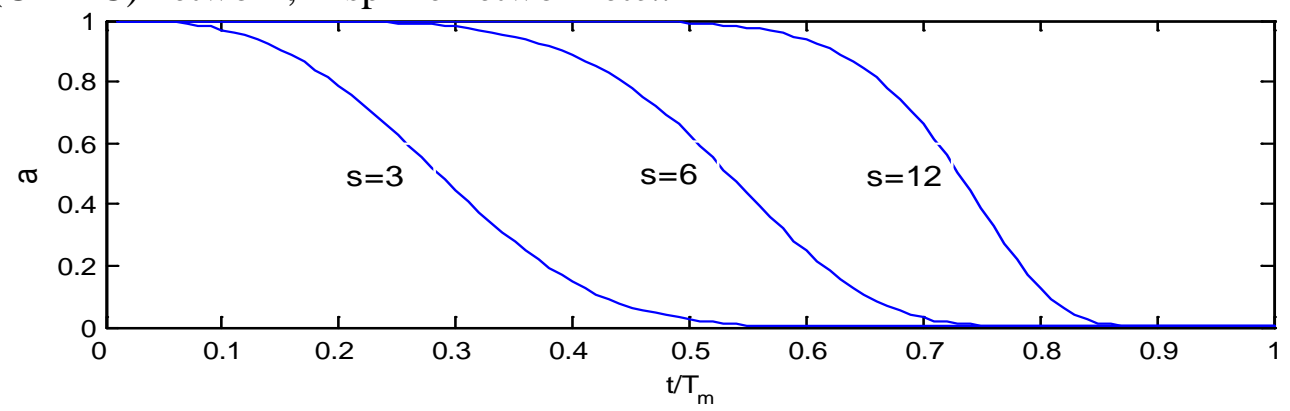

Fig. 1 Variation curve of the function $a$

\section{Training flow of RBF neural network based on adaptive AFSA algorithm}

The realization steps of WNN trained by adaptive AFSA follows as [5]:

1. Initialize the artificial fish swarm size $M$, the initial position of each artificial fish, the vision domain Visual , the step size Step, the crowding degree $\delta$, maximum repeated attempts factor try-number , the maximum number Maxiter of iterations;

2. set the initial iterations times num $=0$, randomly generate $M$ individuals of the artificial fish in the feasible domain of control variables, forming the initial fish swarm, namely produce $M$ group parameters including: the center, the width and connection weight of the network, and these parameters can be taken as the random number in the interval $[-1,1]$.

3. Calculate the fitness of each artificial fish, compare with the state on bulletin board, if better, then assign it to a bulletin board;

4. Calculate the vision demian Visual and step size Step according to the formula (1);

5. Update their position by each artificial fish through the foraging, clustering, pileup and random behavior;

6. Judge whether it has reached the maximum number of iterations, if satisfied, algorithm is terminated the and the results are outputed (i.e., the value on bulletin board); otherwise num +1 , turn to step 3;

7. The optimal solution obtained by improved AFSA algorithm is decoded as the optimal parameters of RBF neural network, which can get the training model of RBF neural network.

\section{Numerical example}

For a nonlinear piecewise function

$$
f(x)= \begin{cases}0.4 x+4 & -10 \leq x \leq-2 \\ 3.2 & -2 \leq x<1 \\ 3.2 e^{-0.5(x-1)} \cos \left(0.6 x^{2}+0.6 x\right) & 1 \leq x \leq 10\end{cases}
$$

Radomly take 481 sample points in $[-10,10]$. The experimental parameter settings: the size of the fish swarm is 40, the maximum number of iterations Maxiter=1000, Try-number=10, Visual =2.95, 
Step $=0.15, \delta=0.618$, according to the experience formula, the number of the nodes in hidden layer can be taken as 15. After the model is trained, Fig.2 and Fig.3 gives the approximate effect and error, the training convergence curve is shown in Fig.4, "---" denotes the approximation curve, "-" denotes the reference curve. Table 1 shows the comparison of approximation performance between two models.

It can be seen from the experiment result, the overall performance of RBF neural network using adaptive AFSA algorithm has a good improvement from the accuracy and convergence, this shows that the global optimization technology based on adaptive AFSA algorithm, can effectively reduce the system error caused by the nonlinear characteristics of the system, improve the performance of RBF neural network approximation.

Table 1 Comparison of approximation result between 2 algorithm

\begin{tabular}{ccc}
\hline Model performance & RBFNN & Adaptive AFSA-RBFNN \\
\hline Approximation MSE & 0.0629 & 0.0165 \\
Training time (s) & 224.1 & 124.6 \\
\hline
\end{tabular}

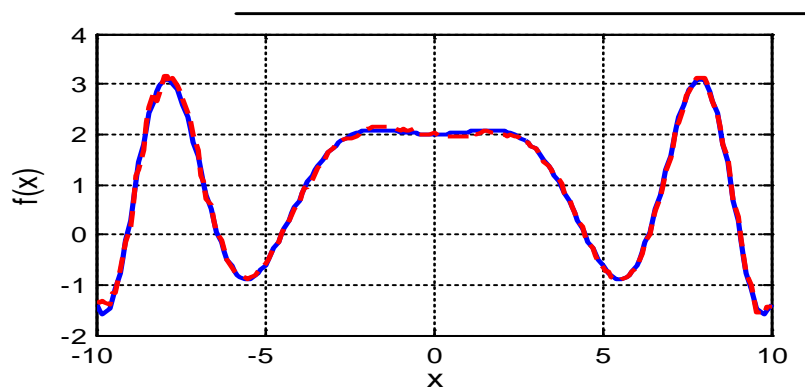

Fig.2 Approximate effect

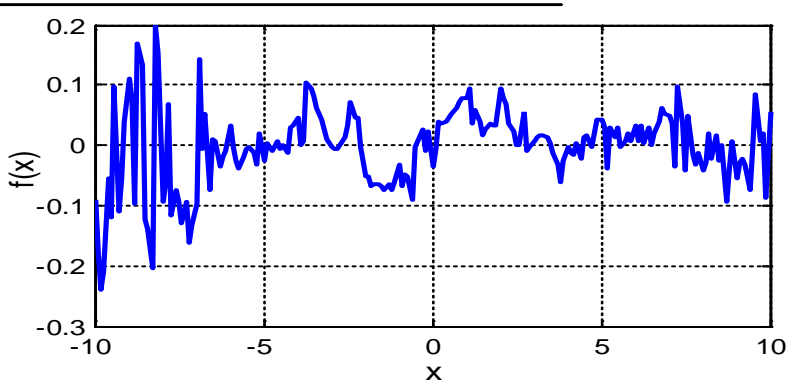

Fig.3 Approximate error

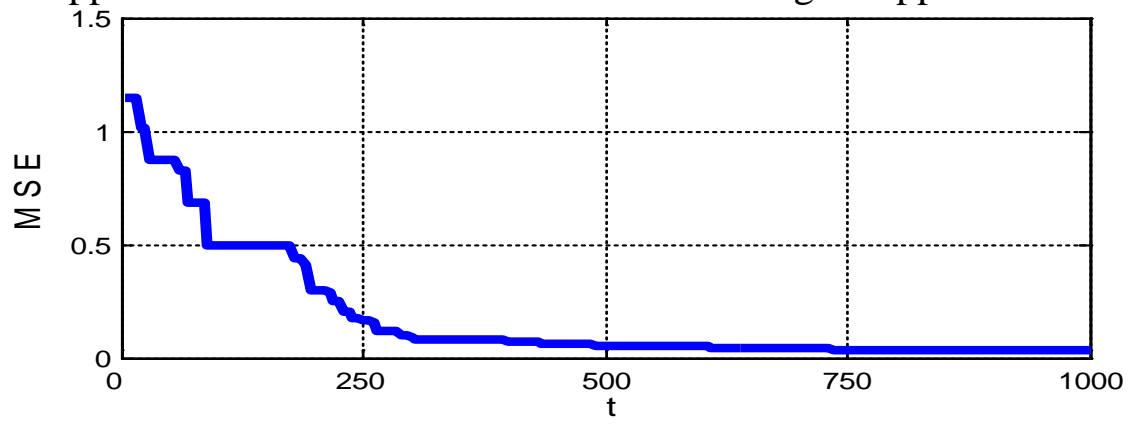

Fig.4 Convergence curve of RBFNN based on adaptive AFSA

\section{Conclusions}

A RBF neural network model based on adaptive AFSA algorithm is proposed. In improved AFS algorithm, the step size of search and the visible domain of the artificial fish can be adjusted in an adaptive form in terms of three stages. Numerical example indicates that the proposed RBF neural network model based on adaptive AFSA algorithm has a good approximation performance for the nonlinear function.

\section{References}

[1] S. Chert, S. A. Billings, P. M. Grant. Recursive hybrid algorithm for nonlinear system identification using radial basis function networks. International Journal of Control, 55(5), (1992), 1051-1070

[2 ] S. Chert, P. M. Crant, C. F. N. Cown. Orthogonal least square algorithm for radial basis function networks. IEEE Transaction on Neural Networks, 2(2), (1991), 302-309 
[3] C. R. Wang, C. L. Zhou, J. W. Ma. An improved artificial fish swarm algorithm and its application in feedforward neural networks. Proceedings of the Fourth International Conference on Machine Learning and Cybernetics, (2005), 2890-2894

[4] X. L. Li, Z. J. Shao, J. X. Qian. An optimizing method based on autonomous animats: fish-swarm algorithm. Systems Engineering Theory \& Practice, 22(11), (2002), 32-38

[5] X. L. Li, J. X. Qian. Studies on artificial fish swarm optimization algorithm based on decomposition and coordination techniques. Journal of Circuits and System, 8(1), (2003), 1-6 